\title{
DERIVATION OF THREE FUNDAMENTAL MASSES AND LARGE NUMBERS HYPOTHESIS BY DIMENSIONAL
}

\author{
ANALYSIS \\ Dimitar Valev \\ Stara Zagora Department, Space Research and Technology Institute, \\ Bulgarian Academy of Sciences, P.O. Box 73, 6000 Stara Zagora, Bulgaria
}

\begin{abstract}
Three mass dimension quantities have been derived by dimensional analysis by means of fundamental constants - the speed of light in vacuum $(c)$, the gravitational constant $(G)$, the Planck constant $(\hbar)$ and the Hubble constant $(H)$. The extremely small mass $m_{1} \sim \hbar H / c^{2} \sim 10^{-33} \mathrm{eV}$ has been identified with the Hubble mass $m_{H}$, which seems close to the graviton mass $m_{G}$. The enormous mass $m_{2} \sim c^{3} /(G H) \sim 10^{53} \mathrm{~kg}$ is close to the mass of the Hubble sphere and practically coincides with the Hoyle-Crvalho formula for the mass of the observable universe. The third mass $m_{3} \sim \sqrt[5]{H \hbar^{3} / G^{2}} \sim 10^{7} \mathrm{GeV}$ could not be unambiguously identified at present time. Besides, it has been found remarkable fact that the Planck mass $m_{P l} \sim \sqrt{\hbar c / G}$ appears geometric mean of the extreme masses $m_{1}$ and $m_{2}$. Finally, the substantial large number $N=\sqrt{c^{5} /\left(2 G \hbar H^{2}\right)} \approx 5.73 \times 10^{60}$ has been derived relating cosmological parameters (mass, density, age and size of the observable universe) and fundamental microscopic properties of the matter (Planck units and Hubble mass). Thus, a precise formulation and proof of Large Numbers Hypothesis ( LNH) has been found.
\end{abstract}

PACS numbers: 06.20.fa, 06.30.Dr, 14.80. -j, 98.80.Es

Keywords: dimensional analysis, mass of the universe, Hubble mass, large numbers hypothesis 


\section{INTRODUCTION}

The Planck mass $m_{P l} \sim \sqrt{\hbar c / G}$ has been introduced in [1] by means of three fundamental constants - the speed of light in vacuum $(c)$, the gravitational constant $(G)$ and the reduced Planck constant $(\hbar)$. Since the constants $c, G$ and $\hbar$ represent three very basic aspects of the universe (i.e. the relativistic, gravitational and quantum phenomena), the Planck mass appears to a certain degree a unification of these phenomena. The Planck mass have many important aspects in the modern physics. One of them is that the energy equivalent of Planck mass $E_{P l}=m_{P l} c^{2} \sim \sqrt{\hbar c^{5} / G} \sim 10^{19} \mathrm{GeV}$ appears unification energy of four fundamental interactions [2]. Also, the Planck mass can be derived by setting it as the mass whose Compton wavelength and gravitational radius are equal [3]. Analogously, formulae for Planck length $l_{P l} \sim 10^{-35} \mathrm{~m}$, Planck time $t_{P l}=l_{P l} / c$ and Planck density $\rho_{P l} \sim 10^{96} \mathrm{~kg} / \mathrm{m}^{3}$ have derived by dimensional analysis. In quantum gravity models, the Planck length is the length scale at which the structure of spacetime becomes dominated by quantum effects.

The Planck mass formula has been derived by dimensional analysis using fundamental constants $c, G$ and $\hbar$. The dimensional analysis is a conceptual tool often applied in physics to understand physical situations involving certain physical quantities [4-8]. It is routinely used to check the plausibility of the derived equations and computations. When it is known, the certain quantity with which other determinative quantities would be connected, but the form of this connection is unknown, a dimensional equation is composed for its finding. In the left side of the equation, the unit of this quantity $q_{0}$ with its dimensional exponent has been placed. In the right side of the equation, the product of units of the determinative quantities $q_{i}$ rise to the unknown exponents $n_{i}$ has been placed $\left[q_{0}\right] \sim \prod_{i=1}^{n}\left[q_{i}\right]^{n_{i}}$, where $n$ is positive integer and the exponents $n_{i}$ are rational numbers. Most often the dimensional analysis has applied in the mechanics and other fields of the modern physics where there are many problems having a few determinative quantities.Many interesting and important problems related to the fundamental constants have been considered in [9-13].

The discovery of the linear relationship between recessional velocity of distant galaxies, and distance $v=H r$ [14] introduces new fundamental constant in physics and cosmology - the famous Hubble constant $(H)$. Even seven years before, Friedman [15] derived his equations from the Einstein field equations [16], showing that the universe might expand at a rate calculable by the equations. The Hubble constant determines the age of the universe 
$H^{-1}$, the Hubble distance $c H^{-1}$, the critical density of the universe $\rho_{c}=3 H^{2} /(8 \pi G) \sim$ $10^{-26} \mathrm{~kg} / \mathrm{m}^{3}$ [17], and other large-scale properties of the universe.

Because of the importance of the Hubble constant, in the present paper we include $H$ in dimensional analysis together with $c, G$ and $\hbar$ aiming to find the new mass dimension quantities $m_{i} \sim \prod_{j=1}^{3} q_{j}^{n_{j}}$, where every triad $q_{1}, q_{2}, q_{3}$ consists of three constants $c, G, \hbar$ or $H$. Thus, the Hubble constant will represent cosmological phenomena in new derived fundamental masses. The attempt to compose a mass dimension quantity by means of the four constants together produces an undetermined system of linear equations and it has been neglected. According to the recent cosmology, the Hubble "constant" slowly decreases with the age of the universe, but there are indications that other constants, especially gravitational and fine structure constants also vary with comparable rate [18-20]. That is why, the Hubble constant could deserve being treated on an equal level with the other three constants used from Planck.

Dirac [18] suggested the Large Numbers Hypothesis $(L N H)$ pointing out that the ratio of the age of the universe $H^{-1}$ and the atomic unit of time $\tau=e^{2} /\left(m_{e} c^{3}\right) \sim 10^{-23} \mathrm{~s}$ is a large number of the order of $10^{40}$. Besides, the ratio of electrostatic $e^{2} / r^{2}$ and gravitational forces $G m_{e} m_{p} / r^{2}$ between proton and electron in a hydrogen atom is of the order of $10^{39}$ and the ratio of mass of the observable universe $M$ and nucleon mass roughly is of the order of $10^{80}$ :

$$
\frac{H^{-1}}{\tau} \sim \frac{e^{2}}{G m_{e} m_{p}} \sim \sqrt{\frac{M}{m_{p}}} \sim N_{D}
$$

where $e$ is the charge of the electron, $m_{e}$ is the electron mass, $m_{p}$ is the proton mass and $N_{D} \sim 10^{40}$ is the Dirac's large number.

Relying on the ratios (1), he proposed that as a consequence of causal connections between macro and micro physical world, gravitational constant $G$ slowly decreases with time whereas mass of the universe increases in result of slow creation of matter. Although the $L N H$ is inconsistent with General Relativity, the former has inspired and continues to inspire a significant body of scientific literature.

Many other interesting ratios have been found approximately relating some cosmological parameters and microscopic properties of the matter. For example, Narlikar [21] shows that the ratio of radius of the observable universe and classical radius of the electron $e^{2} /\left(m_{e} c^{2}\right)$ is of the order of $10^{40}$. Besides, the ratio of the electron mass and Hubble (mass) parameter 
$\hbar H / c^{2}$ approximates to $10^{39}[22]$. Jordan [23] noted that the mass ratio for a typical star and an electron is of the order of $10^{60}$. The ratio of mass of the observable universe and Planck mass is of the order of $10^{61}$ [24]. Peacock [25] points out that the ratio of Hubble distance and Planck length is of the order of $10^{60}$. Finally, the ratio of Planck density $\rho_{P l}$ and recent critical density of the universe $\rho_{c}$ is found to be of the order of $10^{121}$ [26]. Most of these large numbers are rough ratios of astrophysical parameters and microscopic properties of the matter determined with accuracy of the order of magnitude.

\section{DERIVATION OF THREE FUNDAMENTAL MASSES BY DIMENSIONAL ANALYSIS}

A quantity $m_{1}$ having mass dimension could be composed by means of the fundamental constants $c, \hbar$ and $H$ :

$$
m_{1}=k c^{n_{1}} \hbar^{n_{2}} H^{n_{3}}
$$

where $n_{1}, n_{2}$ and $n_{3}$ are unknown exponents to be determined by matching the dimensions of both sides of the equation, and $k$ is dimensionless parameter of an order of magnitude of a unit.

As a result we find the system of linear equations:

$$
\begin{array}{r}
n_{1}+2 n_{2}=0 \\
-n_{1}-n_{2}-n_{3}=0 \\
n_{2}=1
\end{array}
$$

The unique solution of the system is $n_{1}=-2, n_{2}=1, n_{3}=1$. Replacing obtained values of the exponents in equation (2) we find formula (4) for the mass :

$$
m_{H}=m_{1} \sim \frac{\hbar H}{c^{2}}
$$

The recent experimental values of $c, \hbar$ and $H$ are used: $c=299792458 \mathrm{~m} / \mathrm{s}, \hbar=$ $1.054571596 \times 10^{-34} \mathrm{~J} \mathrm{~s}[27]$ and $H \approx 70 \mathrm{~km} / \mathrm{s}$ Mps [28]. Replacing these values in (4) we obtain $m_{1} \sim 2.70 \times 10^{-69} \mathrm{~kg}=1.52 \times 10^{-33} \mathrm{eV}$. This exceptionally small mass coincides 
with the so called "Hubble mass" $m_{H}=\hbar H / c^{2}[29,30]$, which seems close to the graviton mass $m_{G}$ obtained by different methods [31-34]. Evidently, the mass $m_{1}=m_{H} \sim m_{G}$ is in several orders of magnitude smaller than the upper limit of the graviton mass, obtained by astrophysical constraints [35].

From formula (4) we find that the reduced Compton wavelength $\lambda_{1}$ of this mass is equal to the Hubble distance $c H^{-1}$ :

$$
\lambda_{G} \sim \lambda_{1}=\frac{\hbar}{m_{1} c}=c H^{-1} \sim 1.3 \times 10^{26} \mathrm{~m}
$$

The Compton wavelength of graviton (gauge boson of gravity) determines the range of gravitational interaction that appears finite due to of massive graviton. The range of gravity is of the order of magnitude of the Hubble distance, therefore the last gives the size of gravitationally connected (observed) universe for an arbitrary observer.

Analogously, by means of the fundamental constants $c, G$ and $H$, a quantity $m_{2}$ having dimension of a mass could be composed:

$$
m_{2}=k c^{n_{1}} G^{n_{2}} H^{n_{3}}
$$

where $n_{1}, n_{2}$ and $n_{3}$ are unknown exponents to be determined by matching the dimensions of both sides of the equation, and $k$ is dimensionless parameter of an order of magnitude of a unit.

We determine the exponents $n_{1}=3, n_{2}=-1, n_{3}=-1$ by the dimensional analysis again. Replacing the obtained values of the exponents in formula (6) we find formula (7) for the mass $m_{2}$ :

$$
M \sim m_{2} \sim \frac{c^{3}}{G H}
$$

First of all, the formula (7) has been derived by dimensional analysis in [36]. This formula practically coincides with Hoyle formula for the mass of the observable universe $M=c^{3} /(2 G H)[37]$ and perfectly coincides with Carvalho formula [38] for the mass of the observable universe, obtained by totally different approach.

The Hubble sphere is the sphere where the recessional velocity of the galaxies is equal to the speed of the light in vacuum $c$, and according to the Hubble law $v=c$ when $r=c H^{-1}$. Besides, the Hubble sphere coincides with gravitationally connected universe for an arbitrary 
observer. Thus, the Hubble sphere appears a three-dimensional sphere, centered on the observer, having radius $r=c H^{-1}$ and density $\bar{\rho} \approx \rho_{c}$. Evidently, the formula (7) is close to the mass of the Hubble sphere $M_{H}$ :

$$
M=M_{H}=\frac{4}{3} \pi \frac{c^{3}}{H^{3}} \frac{3 H^{2}}{8 \pi G}=\frac{c^{3}}{2 G H}
$$

Replacing the recent values of the constants $c, G$ and $H$ in (7) we obtain $m_{1} \sim 1.76 \times$ $10^{53} \mathrm{~kg}$. Therefore, the enormous mass $m_{2}$ would be identified with the mass of the observable universe $M$.

From formulae (4) and (7) we find a remarkable relation (9):

$$
\sqrt{m_{1} m_{2}}=\sqrt{\frac{\hbar c}{G}}=m_{P l}
$$

Therefore, the Planck mass appears geometric mean of the Hubble mass and the mass of the observable universe. As the physical quantity mass is among the most important properties of the matter, the formula (9) hints at a deep relation of the micro particles and the entire universe.

The third quantity $m_{3}$ having dimension of a mass could be constructed by means of the fundamental constants $G, \hbar$ and $H$ :

$$
m_{3}=k G^{n_{1}} \hbar^{n_{2}} H^{n_{3}}
$$

We determine the exponents $n_{1}=-\frac{2}{5}, n_{2}=\frac{3}{5}, m_{3}=\frac{1}{5}$ by dimensional analysis again. Replacing the obtained values of the exponents in formula (10) we find formula (11) for the mass $m_{3}$ :

$$
m_{3} \sim \sqrt[5]{\frac{H \hbar^{3}}{G^{2}}}
$$

Replacing the recent values of the constants $G, \hbar$ and $H$, the mass $m_{3}$ takes value $m_{3} \sim$ $1.43 \times 10^{-20} \mathrm{~kg} \approx 8.0 \times 10^{6} \mathrm{GeV}$. This mass is a dozen of orders of magnitude lighter than the Planck mass and several orders of magnitude heavier than the heaviest known particles like the top quark $m_{t} \approx 174.3 \mathrm{GeV}$ [39]. On the other hand, the energy $m_{3} c^{2} \sim 8 \times 10^{6} \mathrm{GeV}$ appears medial for the important GUT scale $E_{G U T} \sim 10^{16} \mathrm{GeV}$ and electroweak scale $E_{E W} \sim$ $10^{2} \mathrm{GeV}$. Therefore, the mass/energy $m_{3}$ could not be unambiguously identified at the present time, and it could be considered as heuristic prediction of the suggested approach 
concerning unknown very heavy particle or fundamental energy scale. In the first time these three masses have been derived in [40].

Finally, we would like again to demonstrate the heuristic power of the suggested approach approximately estimating the total density of the universe by dimensional analysis. Actually, a quantity $\rho$ having dimension of a density could be constructed by means of the fundamental constants $c, G$ and $H$ :

$$
\rho=k c^{n_{1}} G^{n_{2}} H^{n_{3}}
$$

where $k$ is a dimensionless parameter of the order of magnitude of a unit.

By the dimensional analysis, we have found the exponents $n_{1}=0, n_{2}=-1, n_{3}=2$. Therefore:

$$
\rho \sim \frac{H^{2}}{G} \approx 7.9 \times 10^{-26} \mathrm{~kg} / \mathrm{m}^{3}
$$

The recent Cosmic Microwave Background $(C M B)$ observations show that the total density of the universe $\bar{\rho}$ is $[41-43]$ :

$$
\bar{\rho}=\Omega \rho_{c} \approx \rho_{c}=\frac{3 H^{2}}{8 \pi G} \sim 10^{-26} \mathrm{~kg} / \mathrm{m}^{3}
$$

Evidently, the density $\rho$ derived by means of the fundamental constants $c, G$ and $H$ coincides with formula (14) for the total density of the universe with an accuracy of a dimensionless parameter of an order of magnitude of a unit. Besides, the formula (13) could be derived by means of other triad of fundamental constants, namely $G$, $\hbar$ and $H$.

\section{DERIVATION OF LARGE NUMBERS HYPOTHESIS}

The Planck mass $m_{P l} \sim \sqrt{\hbar c / G}$ and formulae (4) and (7) for the Hubble mass and mass of the observable universe have been derived by dimensional analysis by means of the fundamental constants $c, G, \hbar$ and $H$. The Planck density $\rho_{P l} \sim c^{5} /\left(\hbar G^{2}\right) \approx 5.2 \times$ $10^{96} \mathrm{~kg} / \mathrm{m}^{3}$, the Planck length $l_{P l} \sim \sqrt{G \hbar / c^{3}} \approx 1.1 \times 10^{-35} \mathrm{~m}$, the Planck time $t_{P l}=$ $l_{P l} / c \sim \sqrt{G \hbar / c^{5}} \approx 5.4 \times 10^{-44} \mathrm{~s}$ and the formula (13) for the total density of the universe also are obtained by dimensional analysis. Taking into account above mentioned formulae and Hubble distance $\mathrm{cH}^{-1}$ and age of the universe $H^{-1}$ we find remarkable ratios (15): 


$$
\sqrt{\frac{M}{m_{H}}}=\frac{M}{m_{P l}}=\frac{m_{P l}}{m_{H}}=\frac{c H^{-1}}{l_{P l}}=\frac{H^{-1}}{t_{P l}}=\sqrt{\frac{\rho_{P l}}{\bar{\rho}}}=\sqrt{\frac{c^{5}}{G \hbar H^{2}}}=N \approx 8.1 \times 10^{60}
$$

These ratios appear very important because they relate cosmological parameters (mass, density, age and size of the observable universe) and the fundamental microscopic properties of the matter (Planck mass, Planck density, Planck time, Planck length and Hubble mass). In recent quantum gravity models, the Planck units imply quantization of spacetime at extremely short range. Thus, the ratios (15) represent connection between cosmological parameters and quantum properties of spacetime. Obviously, the ratios (15) appear a formulation of $L N H$.

As it has been mention in Section 1, the dimensional analysis allows to find unknown quantity with accuracy to dimensionless parameter $k$ of the order of magnitude of unit. Below, we shall recalculate the ratios (15) using exact values of the respective quantities. The exact value of Planck mass could be found from definition of the Planck mass as the mass $m$, whose Compton wavelength $\lambda$ and gravitational (Schwarzschild) radius $r_{S}$ are equal:

$$
\lambda=\frac{\hbar}{m c}=r_{S}=\frac{2 G m}{c^{2}}
$$

Thus, from (16) we find the exact value of Planck mass:

$$
m_{P l}=\sqrt{\frac{\hbar c}{2 G}} \approx 1.53 \times 10^{-8} \mathrm{~kg}
$$

The exact value of Planck length $l_{P l}$ could be found from (16) and (17):

$$
l_{P l}=r_{S}=\sqrt{\frac{2 G \hbar}{c^{3}}} \approx 1.61 \times 10^{-35} \mathrm{~m}
$$

Finally, the exact value of the Planck density is the density of a sphere possessing mass $m_{P l}$ and radius $l_{P l}$ :

$$
\rho_{P l}=\frac{3}{16 \pi} \frac{c^{5}}{\hbar G^{2}} \approx 3.1 \times 10^{95} \mathrm{~kg} / \mathrm{m}^{3}
$$

Taking into account formulae (4), (8), (17), (18) and (19) as well as the Planck time $t_{P l}=l_{P l} / c=\sqrt{2 G \hbar / c^{5}} \approx 3.8 \times 10^{-44} \mathrm{~s}$, Hubble distance $c H^{-1}$, Hubble time ("age of the universe") $H^{-1}$ and total density of the universe $\bar{\rho} \approx \rho_{c}=3 H^{2} /(8 \pi G)$ we find the ratios (20): 


$$
\sqrt{\frac{M}{m_{H}}}=\frac{M}{m_{P l}}=\frac{m_{P l}}{m_{H}}=\frac{c H^{-1}}{l_{P l}}=\frac{H^{-1}}{t_{P l}}=\sqrt{\frac{\rho_{P l}}{\bar{\rho}}}=\sqrt{\frac{c^{5}}{2 G \hbar H^{2}}}=N \approx 5.73 \times 10^{60}
$$

It is worth noting that all ratios (20) are exact. Besides, the large number $N=$ $\sqrt{c^{5} /\left(2 G \hbar H^{2}\right)} \approx 5.73 \times 10^{60}$ is not simply ratio of two quantities but it is a formula expressed by means of the fundamental constants $c, G, \hbar$ and $H$. Thus, the ratios (20) represent exact formulation of the $L N H$ while the ratios (15) are approximate.

The relation (21) could be found from (4), (8), (14) and (19):

$$
v_{0}=\frac{M}{\rho_{P l}}=\frac{m_{H}}{\bar{\rho}}=\frac{8 \pi}{3} \frac{G \hbar}{H c^{2}} \approx 2.8 \times 10^{-43} \mathrm{~m}^{3}
$$

The radius of the sphere having volume $v_{0}$ is $r_{0} \approx 4.1 \times 10^{-15} \mathrm{~m}$, that is of the order of size of the atomic nucleus. Therefore, the formula (21) shows that when the size of the universe was of the order of atomic nucleus its density was close to the Planck density $\rho_{P l}$. Besides, the volume of the recent universe having average density $\bar{\rho} \approx \rho_{c} \sim 10^{-26} \mathrm{~kg} / \mathrm{m}^{3}$ holds matter and energy equivalent to the Hubble (graviton) mass $m_{H}$.

As the large number $N$ is inverse proportional to $H$, the former increases during the expansion. The ratios (20) show that the mass of the observable universe $M$ increases linearly with the cosmological time $H^{-1}$, whereas Hubble (graviton) mass decreases. Besides, the total density of the universe $\bar{\rho} \approx \rho_{c}$ decreases quadratic with cosmological time. However, the time variations of these quantities are negligible:

$$
\frac{\dot{M}}{M}=-\frac{m_{H}}{m_{H}}=-\frac{1}{2} \frac{\dot{\bar{\rho}}}{\bar{\rho}}=\frac{\dot{N}}{N} \sim H \approx 7.3 \times 10^{-11} y^{-1}
$$

In addition, the large number $N$ and Dirac's large number $N_{D}$ are connected by the approximate formula (23):

$$
N_{D} \sim N^{2 / 3}=\sqrt[3]{\frac{c^{5}}{2 G \hbar H^{2}}} \approx 3.2 \times 10^{40}
$$

\section{CONCLUSIONS}

Three mass dimension quantities $m_{i}$ have been derived by dimensional analysis, in addition to the Planck mass $m_{P} \sim \sqrt{\hbar c / G} \approx 2.17 \times 10^{-8} \mathrm{~kg}$. Four fundamental constants - the 
speed of light in vacuum $(c)$, the gravitational constant $(G)$, the reduced Planck constant $(\hbar)$ and the Hubble constant $(H)$ have been involved in the dimensional analysis. The first derived mass dimension quantity $m_{1} \sim \hbar H / c^{2} \sim 10^{-33} \mathrm{eV}$ has been identified with the Hubble mass, which seems close to the graviton mass. The enormous mass $m_{2} \sim c^{3} /(G H) \sim 10^{53} \mathrm{~kg}$ is close to the mass of the Hubble sphere that appears gravitationally connected universe for an arbitrary observer. Besides, this formula practically coincides with the Hoyle-Carvalho formula for the mass of the universe obtained by totally different approach. The identification of the two derived masses reinforces the trust in the suggested approach. It is remarkable that the Planck mass appears geometric mean of the masses $m_{1}$ and $m_{2}$, i.e. $m_{P l}=\sqrt{m_{1} m_{2}}$. The third derived mass $m_{3} \sim \sqrt[5]{H \hbar^{3} / G^{2}} \sim 10^{7} \mathrm{GeV}$ could not be identified unambiguously at present time, and it could be considered as heuristic prediction of the suggested approach concerning unknown very heavy particle or fundamental energy scale.

Besides, the order of magnitude of the total density of the universe has been estimated by means of the suggested approach. Finally, a unique formula for large number $N=\sqrt{c^{5} /\left(2 G \hbar H^{2}\right)} \approx 5.73 \times 10^{60}$ has been derived relating cosmological parameters (mass, density, age and size of the observable universe) and fundamental microscopic properties of the matter (Planck mass, Planck density, Planck time, Planck length and Hubble mass). Thus, a precise formulation and proof of $L N H$ has been found.

\section{REFERENCES}

[1] M. Planck, The Theory of Heat Radiation (New York: Dover Publications, 1959) - translation from German Ed. 1906.

[2] H. Georgi, H.R. Quinn and S. Weinberg, Phys. Rev. Lett. 33, 451 (1974).

[3] P.G. Bergmann, The Riddle of Gravitation (New York: Dover Publications, 1992).

[4] P.W. Bridgman, Dimensional Analysis (New Haven: Yale University Press, 1922).

[5] H.E. Huntley, Dimensional Analysis (New York: Dover Publications, 1967).

[6] R. Kurth, Dimensional Analysis and Group Theory in Astrophysics (Oxford: Pergamon Press, 1972).

[7] R. Bhaskar and A. Nigam, Artificial Intelligence 45, 73 (1990).

[8] G.W. Petty, Software - Practice and Experience 31, 1067 (2001). 
[9] J.M. Levy-Leblond, Riv. Nuovo Cim. 7, 187 (1977).

[10] M.J. Duff, Preprint: arxiv:hep-th/0208093 (2002).

[11] M.J. Duff, L.B. Okun and G. Veneziano, J. High En. Phys. Issue 03, 023 (2002).

[12] J.D. Barrow, The Constants of Nature: From Alpha to Omega (London: Jonathan Cape, 2002).

[13] H. Fritzsch, The Fundamental Constants, a Mystery of Physics (Singapore: World Scientific, 2009).

[14] E. Hubble, Proc. Nat. Acad. Sci. 15, 168 (1929).

[15] A. Friedman, Z. Physik 10, 377 (1922).

[16] A. Einstein, Annalen der Physik 354, 769 (1916).

[17] P.J. Peebles, Physical Cosmology (Princeton: Princeton University Press, 1971).

[18] P.A.M. Dirac, Nature 139, 323 (1937).

[19] Y. Wu and Z. Wang, Phys. Rev. Lett. 57, 1978 (1986).

[20] J.K. Webb et al., Phys. Rev. Lett. 87, id. 091301 (2001).

[21] J.V. Narlikar, The Structure of the Universe (Oxford: Oxford University Press, 1977).

[22] A. Cetto, L. de la Pena and E. Santos, Astron. Astrophys. 164, 1 (1986).

[23] P. Jordan, Die Herkunft der Sterne (Stuttgart: Wiss.Verlagsges., 1947).

[24] V.E. Shemi-zadeh, Preprint: arXiv:gr-qc/0206084 (2002).

[25] J.A. Peacock, Cosmological Physics (Cambridge: Cambridge University Press, 1999).

[26] A.Y. Andreev and B.V. Komberg, Astron. Rep. 44, 139 (2000).

[27] P. Mohr and B. Taylor, J. Phys. Chem. Ref. Data 28, 1713 (1999).

[28] J.R. Mould et al., Astrophys. J. 529, 786 (2000).

[29] I. Maor and R. Brustein, Phys. Rev. D 67, id. 103508 (2003).

[30] J.P. Gazeau and F. Toppan, Class. Quantum Grav. 27, id. 025004 (2010).

[31] J.F. Woodward, R. J. Crowley and W. Yourgrau, Phys. Rev. D 11, 1371 (1975).

[32] S.S. Gershtein, A.A. Logunov and M.A. Mestvirishvili, Dokl. Phys. 43, 293 (1998).

[33] D. Valev, Aerospace Res. Bulg. 22, 68(2008); Preprint: arxiv:hep-ph/0507255.

[34] M.E. Alves, O.D. Miranda and J. C. de Araujo, Preprint : arxiv:0907.5190 (2009).

[35] A.S. Goldhaber and M.M. Nietto, Phys. Rev. D 9, 1119 (1974).

[36] D. Valev, Compt. Rend. Acad. Bulg. Sci. Spec. Issue, 233 (2009); Preprint: arXiv:0909.2726.

[37] F. Hoyle, in Proc. 11th Solvay Conference in Physics, Ed. R. Stoops, Brussels, Belgium (1958). 
[38] J.C. Carvalho, Int. J. Theor. Phys. 34, 2507 (1995).

[39] M. Mangano and T. Trippe, Europ. Phys. J. C 15, 385 (2000).

[40] Valev D., Preprint : vixra:1103.0096 (2011).

[41] A. Balbi et al., Astrophys. J. 545, L1 (2000).

[42] P. de Bernardis et al., Nature 404, 955 (2000).

[43] D.N. Spergel et al., Astrophys. J. Suppl. Series 148, 175 (2003). 